\title{
Erratum to: Variable-Internal-Stores models of microbial growth and metabolism with dynamic allocation of cellular resources
}

\author{
Olga A. Nev ${ }^{1}$ - Hugo A. van den Berg ${ }^{2}$
}

Published online: 2 August 2016

(C) Springer-Verlag Berlin Heidelberg 2016

\section{Erratum to: J. Math. Biol. DOI 10.1007/s00285-016-1030-4}

In the original publication of the article the symbol Phi ' $\phi$ ' should be changed to symbol Psi ' $\psi$ ' in Table 1 under the section "Unscaled stoichiometric coefficients", line 2 , column 1 .

The original article has been updated to reflect the above change.

The online version of the original article can be found under doi:10.1007/s00285-016-1030-4.

$凶$ Olga A. Nev

O.Nev@warwick.ac.uk

Hugo A. van den Berg

hugo@maths.warwick.ac.uk

1 Warwick Analytical Sciences Centre, University of Warwick, Coventry CV4 7AL, UK

2 University of Warwick, Coventry CV4 7AL, UK 